\title{
Entre pratique et discours : les villes de la région parisienne face au secret au début du $\mathrm{XV}^{\mathrm{e}}$ siècle
}

\author{
Pierre-Henri Guittonneau
}

\section{(2) OpenEdition}

\section{Journals}

Édition électronique

URL : http://journals.openedition.org/questes/709

DOI : $10.4000 /$ questes.709

ISSN : 2109-9472

Éditeur

Les Amis de Questes

Édition imprimée

Date de publication : 15 avril 2009

Pagination : 12-24

ISSN : 2102-7188

\section{Référence électronique}

Pierre-Henri Guittonneau, «Entre pratique et discours : les villes de la région parisienne face au secret au début du XVe siècle », Questes [En ligne], 16 | 2009, mis en ligne le 01 janvier 2014, consulté le 02 mai 2019. URL : http://journals.openedition.org/questes/709 ; DOI : 10.4000/questes.709 


\title{
Entre pratique et discours : les villes de la région parisienne face au secret au début du $X V^{\mathrm{e}}$ siècle
}

\author{
Pierre-Henri GUITTONNEAU
}

Il est de coutume de dire que le secret en politique a été longtemps laissé à la petite histoire et tenu en marge de la production historique scientifique, car sulfureux ${ }^{1}$. La période de la guerre civile entre Armagnacs et Bourguignons n'a pas échappé à ce phénomène. Sa postérité littéraire suffit à le démontrer. À côté des Lorenzaccio et des Cromwell, le XIX ${ }^{\mathrm{e}}$ siècle a accouché de drames historiques, récritures simplifiées et romanesques des chroniques du $\mathrm{XV}^{\mathrm{e}}$ siècle où, à côté de la folie de Charles VI et de la rivalité entre Louis d'Orléans et Jean Sans Peur, la pratique du secret est omniprésente $^{2}$.

Ces pièces, certes mineures, sont à ranger, malgré tout, parmi les très nombreuses œuvres relatives à l'histoire $\mathrm{du} \mathrm{XV}^{\mathrm{e}}$ siècle qui ont paru au même moment ${ }^{3}$. La plupart de ces dernières, surtout des éditions de chroniques, ont constitué, et constituent toujours, des corpus de sources pour des historiens qui s'intéressent d'un point de vue politique à cette période. Dans ces études

1 MONIER, Frédéric, «Le secret en politique, une histoire à écrire », Matériaux pour l'histoire de notre temps, 58 (2000), p. 3-8. Dressant un bilan historiographique de la question, l'auteur fait référence à de nombreuses études tant d'historiens modernistes et contemporanéistes que de sociologues, philosophes ou encore psychanalystes.

${ }^{2}$ Parmi les quelques pièces consacrées à cette période, il en est une - Jean Sans Peur, drame historique en cinq actes - qui élève véritablement le secret au rang d'acteur tant il est omniprésent sous la forme de complots, de rumeurs et d'amours cachées. GUILLEM, Gaston, Jean-Sans-Peur, drame historique en cinq actes, Paris, E. Dentu, 1875.

${ }^{3}$ Il s'agit notamment des éditions de chroniques placées sous le patronage de la Société de l'Histoire de France. 
souvent majeures, le secret ne semble pas avoir trouvé toute sa place ${ }^{4}$. C'est assez récemment, semble-t-il, que les médiévistes s'en sont emparé, sans pour autant en faire une synthèse générale servant à l'histoire politique.

En effet, le secret suscite l'intérêt des historiens par le biais de la communication et de l'information, au sens politique ou technique et jurisprudentiel. Un colloque récent a notamment mis en valeur le rôle joué par la divulgation des informations dans les sociétés, que cette divulgation soit le fait des gouvernants ou des gouvernés ${ }^{5}$. Le secret est alors présenté comme un outil politique et aussi un élément sinon constitutif du moins essentiel du lien social $^{6}$. Or, dans un contexte de guerre civile où l'espace privé et l'espace public se brouillent, où le politique et les relations interpersonnelles représentent un enjeu majeur pour deux partis rivaux en quête du pouvoir, la pratique du secret s'impose dans la multiplicité de ses formes. Cette pratique est si présente, si menaçante parfois pour les sociétés, et notamment les sociétés urbaines, qu'elle voit se constituer un discours moral visant à la condamner en tant que facteur de déséquilibre du corps social. C'est de cela dont je vais parler en prenant à mon compte la définition du secret de Georg Simmel à savoir une « limitation de la connaissance réciproque », incluant le

\footnotetext{
${ }^{4}$ On peut citer, entre autres, le livre de Bernard Guenée consacré au meurtre de Louis d'Orléans : GuENEE, Bernard, Un Meurtre, une société. L'assassinat du duc d'Orléans, 23 novembre 1407, Paris, Gallimard, 1992.

5 Boudreau, Claire, Fianu, Kouky, Gauvard, Claude et Hebert, Michel (éds), Information et société en Occident à la fin du Moyen Âge, Actes du colloque international tenu à l'Université du Québec à Montréal et à l'Université d'Ottawa (9-11 mai 2002), Paris, Publications de la Sorbonne, 2004.

${ }^{6}$ Cette impression correspond aux nombreuses études consacrées au secret, notamment à des études de sociologie qui insistent sur le fait qu' «il n'y a pas d'interaction sans une forme ou l'autre de secret ou de révélation ». (PETITAT, André, «Introduction », dans André Petitat (dir.), Secret et lien social, Paris, L’Harmattan, 2000, p. 7).
} 
mensonge, le non-dit ou encore la connivence, afin de percevoir les relations entre le secret et les sociétés urbaines de la région parisienne ${ }^{7}$.

Le choix de cet espace relève de sa place dans les épisodes de la guerre civile dont un grand nombre avait pour objectif la conservation ou la conquête de la capitale par un des deux partis, principalement de 1407 à 1419, du meurtre de Louis d'Orléans à celui de Jean Sans Peur. Le corpus de sources consulté est composé surtout de chroniques mais aussi de registres de délibération et de comptes de commune. Ces documents indiquent soit de manière détaillée soit de façon plus laconique les enjeux du secret dans les villes de la région parisienne. Ils permettent ainsi de distinguer les formes du secret les plus courantes à cette période et les moyens divers de s'en prémunir. Cette question sera ici abordée sous le biais de la révélation et de la publicité du secret.

\section{Le secret dans l'espace public : les formes du secret}

Les mots employés dans les sources pour nommer le secret ne sont pas légion. Le lecteur de Michel Pintoin, le Religieux de Saint-Denis, se rend rapidement compte que le mot « secret »n'est quasiment jamais employé pour lui-même. Hormis dans l'expression «secrets du roi », on ne le rencontre presque que sous ses formes d'adjectif et d'adverbe ; c'est dire qu'on l'utilise avant tout pour qualifier des actions, des attitudes ou des objets : « libelles secrets », «assemblées secrètes », roi enlevé « secrètement ${ }^{8}$. Quand

\footnotetext{
${ }^{7}$ Je reprends ici l'analyse d'André Petitat d'un chapitre de la Soziologei de Georg Simmel, publié en français et séparément en 1945 (SIMMEL, Georg, Secret et sociétés secrètes, trad. fr., Strasbourg, Circé, 1991). PETITAT, André, art. cit., p. 7.

${ }^{8}$ Chronique du Religieux de Saint-Denys, contenant le règne de Charles VI de 1380 à 1422, Louis-François Bellaguet (éd. et trad.), Paris, éditions du Comité des travaux historiques et scientifiques, 6 vol., rééd. 1994, t. 3, p. 191, t. 5, p. 173 et t. 4, p. 183 . La
} 
l'attention se porte plus largement sur l'idée du secret, sur ce qui est séparé et caché, le vocabulaire se diversifie avec un éventail d'expressions comme «à l'insu », « dissimulation », « à la dérobée », « mensonge », « trahison » ou encore $«$ surprise ${ }^{9}$. Ce constat appelle quelques commentaires.

La diversité de la terminologie pousse en effet à envisager le secret dans la multiplicité de ses formes. Ces emplois du vocabulaire contribuent aussi indirectement à rappeler qu'il n'est pas une donnée historique, mais un invariant de l'espace politique qu'il s'agit peut-être plus que d'autres phénomènes de caractériser et de contextualiser ${ }^{10}$. Enfin l'utilisation de ces termes et la présentation des pratiques du secret dans les chroniques renvoient à une caractéristique de ce dernier. À l'exception du secret absolu impossible à appréhender, le secret se cache en même temps qu'il se révèle. Il cache son contenu et révèle son existence ; or les chroniques, plus que d'autres sources, s'il leur arrive de présenter les secrets ainsi, révèlent également et leur existence et leur contenu. C'est sans doute dû au fait que les auteurs écrivent après le dévoilement des secrets qui ont souvent une valeur performative sur le court terme : prendre une ville ou monter une conspiration. Les secrets sont donc connus une fois percés ou quand leur objectif est atteint. Par conséquent, il peut être utile de faire la part des différentes formes du secret tel qu'ils apparaissent dans les sources, à savoir au moment de leur publicité.

Avant de passer aux secrets vraiment liés à la guerre civile, il convient peut-être de mesurer la place du secret politique dans la société, et notamment

remarque est la même pour le vocabulaire latin qu'emploie Michel Pintoin. On trouve ainsi les adverbes clam, occultius ou encore l'adjectif secretus.

${ }^{9}$ Ibid., t. 3, p. 11 et p. 735 , t. 4, p. 507, t. 6, p. 171, t. 4, p. 449.

${ }^{10}$ DEWERPE, Alain, Espion. Une anthropologie historique du secret d'État contemporain, Paris, Gallimard, 1994, p. 11 : «Une histoire du secret est bien problématique parce qu'elle met aux prises un invariant de l'espace politique avec les conditions spécifiques de possibilité de ses usages ». 
la société parisienne. Plusieurs indices, mis en exergue par un travail récent, tendent à prouver que cette dernière, animée d'une volonté de savoir et d'un intérêt pour la chose publique, a accès à un savoir auquel il n'est pas prévu qu'elle accède ${ }^{11}$. Ce savoir, ce sont les «secrets du roi » ${ }^{12}$. Michel Pintoin mentionne à plusieurs reprises ses sources : magistrats qui instruisent des enquêtes, "gens de la cour bien informés », conseillers du roi voire des princes participant à des conférences secrètes ${ }^{13}$. Autre exemple, des lettres de rémission sont accordées à des hommes condamnés pour avoir vitupéré les choix politiques ou diplomatiques du roi $^{14}$. Il n'est pas possible de connaître l'ampleur des conversations informelles qui ont contribué à la publicité de ces secrets, mais les divers témoignages prouvent l'omniprésence d'un personnel de valets, de clercs, de secrétaires, de médecins qui jouent un véritable rôle d'intermédiaires ${ }^{15}$. Il est d'ailleurs remarquable qu'en 1408, à l'idée que Jean Sans Peur revienne à Paris auréolé de son triomphe contre les Liégeois, les princes du sang et la reine, réunis en « conseils secrets » se promettent de ne

${ }^{11}$ NovaK, Veronika, «La source du savoir. Publication officielle et communication informelle à Paris au début du XV $\mathrm{X}^{\mathrm{e}}$ siècle », dans Claire BoudREAU, Kouky FIANU, Claude GAUVARD et Michel HEBERT (éds.), Information et société en Occident..., op. cit., p. 151163. Les lignes qui suivent sont, entre autres, inspirées de cet article. L'auteur a aussi publié un ouvrage que je n'ai pu consulter mais qui, pour la question, semble riche d'enseignements. Eadem, Nouvelles, pouvoir et société. La circulation des informations à Paris à la fin du Moyen Age, Budapest, Gondola-Infonia, 2007.

${ }^{12}$ Chronique du Religieux de Saint-Denys,..., op. cit., t. 5, p. 23. Dans ce passage, ce sont quelques cabochiens, ivres semble-t-il, qui jettent à l'eau un secrétaire du roi qu'ils accusaient d'avoir révélé aux ennemis les secrets du roi en 1412.

${ }^{13}$ Ibid., t. 4, p. 5, t. 5, p. 33 et t. 4, p. 701.

${ }^{14}$ Douet D’ARCQ, Louis (éd.), Choix de pièces inédites relatives au règne de Charles VI, Paris, Société de l'Histoire de France, 1863-1864, t. 1, p. 99-100 et p. 153.

${ }^{15}$ NovAK, Veronika, art. cit., p. 158. L'auteur cite notamment un exemple trouvé dans le journal de Nicolas de Baye, greffier du Parlement. Ce dernier se plaint de la présence aux séances de la Chambre des enquêtes de «vallés et gens estranges » qui font alors de « grandes buveries » et peuvent apprendre les « secrets de la Court ( (Journal de Nicolas de Baye, greffier du Parlement de Paris 1400-1417, Alexandre TuETEY (éd.), 2 vol., Paris, Société de l'Histoire de France, 1885-1888, t. 1, p. 90. 
rien révéler à personne pas même aux officiers royaux ${ }^{16}$. C'est dire la conscience qu'ils ont de l'existence de canaux informels d'informations auxquels ils peuvent eux-mêmes participer. C'est dire aussi la conviction partagée par sans doute tous les gouvernants de contrôler et gérer la communication. Ainsi, à Mantes, le dimanche 10 octobre 1417, les échevins trouvent une «cedulle qui a este trouve atachee en luys de maison de ville ${ }^{17}$. Avant d'en faire lecture publique, le conseil décide de l'apporter au bailli, au capitaine et au conseil du roi. La communication d'une information relève alors vraiment du Bien Commun et d'une gestion minutieuse d'une parole publique et politique ${ }^{18}$. Or, dans le contexte de la guerre civile, la gestion de cette parole entre dans un jeu politique qui cherche à influencer ou effrayer la société pour conquérir et conserver un pouvoir.

En effet dans les chroniques, les formes du secret, si elles sont multiples, ont aussi pour point commun de faire partie des moyens d'actions utilisés par les Armagnacs et les Bourguignons. Sans qu'on puisse parler d'une sorte de stratégie globale du secret, force est de constater que toutes ces formes ont joué un rôle déterminant dans les villes et sur les sociétés. Projet préparé et dissimulé tant que son objectif n'est pas atteint, projet dissimulé mais percé ou bien projet imputé à autrui, le secret est un facteur aggravant de la fièvre obsidionale caractérisant les sociétés urbaines pendant la guerre

${ }^{16}$ Chronique du Religieux de Saint-Denys,..., op. cit., t. 3, p. 181.

${ }_{17}^{17}$ Mantes-la-Jolie, archives municipales, BB 2, fo $181 \mathrm{v}^{\circ}$ (mardi 5 octobre 1417).

18 MonNET Pierre, «Courriers et messages : un réseau de communication à l'échelle urbaine dans les pays d'Empire à la fin du Moyen Âge », dans Claire BoudREAU, Kouky FIANU, Claude GAUVARD et Michel Hebert (éds.), Information et société..., op. cit., p. 284. 
civile $^{19}$. Il est à parier que, soupçonné ou réel, il vient renforcer une méfiance déjà présente dans une société divisée en partis antagonistes.

La chronique de Michel Pintoin montre qu'il n'y a pas un temps du secret suivi d'un temps où il serait absent. Les diverses formes de ce dernier courent tout au long des chapitres : guet-apens, prises inattendues de villes, complots, trahisons ponctuent régulièrement le récit. Les raisons en sont simples. Malgré les trêves, les rancoeurs demeurent ${ }^{20}$. En temps de lutte ouverte, les embuscades armées sont relativement constantes et, à Paris, le parti qui n'est pas au pouvoir tente d'en prendre le contrôle. Ainsi, en 1411, la prise du pont de Saint-Cloud par les partisans du duc d'Orléans provoque-telle la stupeur de l'entourage du Dauphin, tant personne ne s'y attendait ${ }^{21}$. Les conditions semblent être presque toujours réunies pour recourir au secret. Ces remarques faites, il convient cependant de noter que certains moments semblent avoir été plus propices que d'autres à son utilisation.

C'est le cas des périodes précédant ou succédant immédiatement la prise de Paris par un des deux partis. La finalité du secret est alors double :

19 À titre d'illustration, Yves-Marie Bercé, pour l'époque moderne, a montré que « les comploteurs en choisissant la clandestinité s'évadaient [...] des normes de sociabilité de leur temps et inspiraient d'autant plus l'inquiétude, la répulsion, le rejet », BERCE, YvesMarie, "Introduction », dans Complots et conjurations dans l'Europe moderne, Actes du colloque international organisé par l'Ecole française de Rome, l'I.R.C.O.M. de l'Université de Paris-Sorbonne et le Dipartimento di storia moderna et contemporanea dell'Università degli studi di Pisa, Rome, 30 septembre-2 octobre 1993, Yves-Marie BERCE et Elena FASANO GUARINI (dir.), Rome, École française de Rome, 1996 (Collection de l'École française de Rome, 220), p. 2.

${ }^{20}$ En témoigne ailleurs, dans le Journal du Bourgeois de Paris, le ton de l'auteur qui, de manière assez constante, est fort acerbe à l'égard des Armagnacs. Parmi les expressions employées, on trouve notamment : «faulx bandez Armignaz ». (Journal d'un Bourgeois de Paris de 1405 à 1449, Colette BEAune (éd.), Paris, Le Livre de Poche, p. 12).

${ }^{21}$ Chronique du Religieux de Saint-Denys..., op. cit., t. 4, p. 509-511. Le messager, porteur de cette annonce, prié d'apporter des détails met en cause le capitaine du lieu, beau-frère d'un partisan du duc, qui aurait facilité, sans doute contre espèces sonnantes et trébuchantes, l'introduction secrète des assaillants dans la tour protégeant le pont. 
préparer le succès du retour d'un parti et discréditer celui qui est chassé. Soldée par un échec, la conspiration de Nicolas d'Orgemont, président de la Chambre des Comptes, est là pour en attester. Il s'agissait de provoquer le retour de Jean Sans Peur en 1416 en assassinant les principaux notables armagnacs de la ville ${ }^{22}$. L'arrivée au pouvoir d'un nouveau parti voit aussi l'espace public être investi d'une autre forme de secret, secret supposé ou projet imputé à l'adversaire. De pratique, le secret devient alors chef d'accusation. On recherche la disqualification morale d'autrui et la justification de ses propres actes, parfois accomplis sous le sceau du secret. Il en va ainsi de la justification du meurtre de Louis d'Orléans prononcée devant le roi au nom de Jean Sans Peur et de celle prononcée pour laver la mémoire de la victime à deux moments différents de l'année 1408. Parmi les accusations faites à l'un et à l'autre, l'usage du secret se trouve en bonne place. Du côté bourguignon, on dénonce les crimes de sorcellerie et de lèsemajesté de Louis d'Orléans, coupable d'avoir secrètement ensorcelé Charles $\mathrm{VI}^{23}$. Du côté orléanais, après s'être indigné des «libelles mensongers » qui accusent le prince assassiné, on rappelle que ce dernier le fut dans la surprise d'un guet-apens avant d'être pleuré par un Jean Sans Peur pourtant lié à lui par un traité d'amitié contracté peu de temps auparavant ${ }^{24}$. À une autre échelle, lors des révoltes de 1413 et 1418, le mot «trahison », résumant bien les mauvaises intentions que l'on prête à son ennemi, est fréquemment utilisé. Il permet de discréditer des ennemis. Ainsi le prévôt de Paris, Pierre des Essars, est-il décapité en 1413 notamment pour avoir voulu trahir le roi ${ }^{25}$. Cette

\footnotetext{
${ }^{22}$ Ibid., t. 5, p. 3-11.

${ }^{23}$ Ibid., t. 3, p. 757-759.

${ }^{24}$ Ibid., t. 4, p. 115 et p. 111 . On retrouve peu ou prou les mêmes accusations après le meurtre de Jean Sans Peur à Montereau en septembre 1419.

${ }^{25}$ Ibid., t. 5, p. 75-77.
} 
utilisation fait se côtoyer un certain type de secret avec la rumeur, quoique l'un et l'autre ne puissent se confondre. La révélation du secret supposé dans ce cas a les mêmes effets qu'une rumeur: créer un trouble, identifier un danger pour le contenir ou le détruire ${ }^{26}$.

La pratique du secret est donc une activité assez courante à cette période, activité dont parfois la publicité doit, au même titre que la réussite d'une conspiration ou d'une embuscade, contribuer à donner ou assurer le pouvoir à ceux qui en sont responsables. Toutefois, participant à la longueur du conflit, elle se voit aussi condamnée moralement. Chez Michel Pintoin, la France, mère désespérée, se plaint que ses enfants portent partout la dévastation par la guerre et par la trahison ${ }^{27}$. Chez Jean Gerson, la Dissimulation dialogue avec la Sédition et sème en tous lieux le mensonge, le «barat rapineux ${ }^{28}$. C'est dire que le secret, dans l'étendue de ses formes, constitue, durant la guerre civile, non seulement un ressort politique mais aussi psychologique, dont on cherche à se préserver pour cette raison même.

\section{Le secret dans l'espace public : la prévention contre le secret}

Pour se prémunir contre le secret, diverses mesures sont prises dans les villes. Ces réponses aux menaces portées par le secret pendant la guerre civile semblent être commandées par une de ses caractéristiques, à savoir qu'il se

\footnotetext{
${ }^{26}$ La rumeur fait partie, de manière éclatante, de ces facteurs qui ont aggravé la fièvre obsidionale des Parisiens pendant cette période de troubles. Colette Beaune affirme que «les habitants de Paris submergés de réfugiés inquiets voient leurs rythmes habituels perturbés et [que] cette semi oisiveté mal vécue les pousse à la rumeur »; cette rumeur a bel et bien un rôle souvent déterminant dans le mécanisme de la violence en 1413 et 1418 . BeAune, Colette, «La rumeur dans le Journal du Bourgeois de Paris », dans La Circulation des nouvelles au Moyen Âge, XXIV congrès de la S.H.M.E.S., Avignon, juin 1993, Paris, 1994, p. 192.

${ }^{27}$ Chronique du Religieux de Saint-Denys..., op. cit., t. 6, p. 171.

${ }^{28}$ GERSOn, Jean, CEuvres complètes, t. 7 : L'CEuvre française. [2] Sermons et discours en français, Mgr Palémon Glorieux (éd.), Paris-Tournai-Rome, Desclée, p. 1153.
} 
cache et se révèle en même temps. La prévention commence par la connaissance et il sera ici question de ceux qui permettent de connaître l'existence voire le contenu d'un secret.

Il convient tout d'abord d'isoler les termes qui désignent ces personnes puis de considérer leur action. Les textes font apparaître des gens qui ne sont pas nommés, si ce n'est par le pronom « on » ou par des périphrases comme « untel "avait appris de bonne part" » ${ }^{29}$. Soit le « on » désigne ici une sorte de rumeur qu'il appartient ensuite de vérifier soit il s'agit d'informateurs ou d'officiers tels des sergents qui ont l'habitude d'enquêter. Il y a également ceux que le Bourgeois de Paris nomme des «espies », des espions qui étaient «résidants et demeurants à Paris » ${ }^{30}$. Enfin ce sont les hérauts qui apparaissent dans un contexte militaire en épiant les positions de l'ennemi ${ }^{31}$. Plus que le vocabulaire qui les désigne, les actions de ces hommes nous intéressent ici.

En 1411, après que les ducs d'Orléans et de Bourgogne se sont lancés leur défi, le conseil du roi décide de prendre des mesures de sécurité à Paris. On recherche alors «certaines gens qu'on [...] avait désignés comme ayant conspiré contre la ville et comme ayant menacé d'y introduire secrètement les troupes du duc d'Orléans $»^{32}$. En 1416, si Nicolas d'Orgemont et ses compagnons sont arrêtés par le Prévôt de Paris, c'est que ce dernier a appris que des hommes armés se réunissaient autour de «partisans dévoués » de Jean Sans Peur ${ }^{33}$. Dans les deux cas, les informateurs désignent avant tout des

${ }^{29}$ Chronique du Religieux de Saint-Denys..., op. cit., t. 5, p. 3.

${ }^{30}$ Journal d'un Bourgeois de Paris..., op. cit., p. 99. Une note de l'éditrice indique que les réseaux d'espionnage se développent avec les guerres du $\mathrm{XV}^{\mathrm{e}}$ siècle. J'ai trouvé trace de ces hommes pour quelques villes - Melun et Meulan — mais ils sont ici laissés de côté car ils sont mentionnés pour une période ultérieure à celle que je me suis fixée.

${ }^{31}$ Ibid., p. 213. Il ne s'agit pas de faire une classification ici, mais de mettre en lumière les termes employés.

${ }^{32}$ Chronique du Religieux de Saint-Denys..., op. cit., t. 4, p. 449.

${ }^{33}$ Ibid., t. 6, p. 5. 
hommes suspects qui font ouvertement partie d'une des deux factions. Dans le premier cas, on arrête ceux qui pourraient être tentés de refaire un complot. Dans le second, on arrête des hommes soupçonnés de préparer quelque chose qui, dans ce cas, n'est dévoilé qu'après l'arrestation. Ces deux exemples montrent donc que les informateurs semblent se fonder sur une connaissance assez précise des hommes qu'ils dénoncent et de leurs activités présentes et passées, parfois assumées publiquement comme cela doit être le cas des clients des chefs de parti et d'officiers du roi $^{34}$. À ces réputations s'ajoutent des informations obtenues grâce aux relations de voisinage. Le Bourgeois de Paris note aussi que les «espies » font arrêter leurs propres voisins ${ }^{35}$. On voit bien là que le contexte de guerre civile contribue à brouiller les espaces privé et public ainsi que les relations quotidiennes des Parisiens.

Cette identification des personnes dangereuses ou susceptibles de l'être permet de prendre quelques mesures de prévention. Trois d'entre elles seront évoquées ici. La première a déjà été entrevue: en 1411, on arrête des personnes à l'idée qu'elles pourraient préparer un complot. La deuxième mesure est celle prise en 1417 par le connétable Bernard d'Armagnac qui interdit tout conciliabule secret au moment même où les Bourguignons sont aux portes de la capitale. La troisième disposition consiste à bannir des personnes appartenant au parti contraire. S'il s'agit ici d'actions humiliantes, puisque cela équivaut à une mort sociale, c'est aussi une manière de se préserver des conspirations. En 1417, le connétable bannit aussi des

\footnotetext{
${ }^{34}$ Les récits des violences de 1413 et de 1418 , de même que celles des prises de pouvoir par l'un des deux pouvoirs, témoignent — entre beaucoup d'autres indices — de cette publicité des opinions. Les uns quittent la ville, tel Nicolas de Baye en 1418, les autres restent et risquent parfois leur vie, tels les nombreux notables armagnacs tués le 12 juin (respectivement : Chronique du Religieux de Saint-Denys,.., op. cit., t. 6, p. 235 et p. 246). ${ }^{35}$ Chronique du Religieux de Saint-Denys,..., op. cit., t. 6, p. 133. Cette disposition fait partie d'un ensemble de mesures prises par les Armagnacs pour contenir toute tentative de la part de partisans de Jean Sans Peur de prendre Paris.
} 
parlementaires réputés bourguignons « pour maintenir la ville de Paris en plus grant seurté $»^{36}$. Il s'agit donc de protéger une population et un espace contre un possible complot ou la prise de la ville, ce qui n'est pas un souci exclusif à Paris.

À Mantes aussi, le conseil de ville prend des dispositions significatives. En septembre 1418, les clefs des portes, jusque-là réparties entre quatre échevins, sont de manière exceptionnelle confiées à un seul homme, alors que Paris vient de tomber aux mains des Bourguignons et que les Armagnacs prennent plusieurs villes voisines de la capitale ${ }^{37}$. Ce changement intervient en même temps que celui des portiers et surtout du maire, un mois plus tôt que prévu. Si aucune explication n'est donnée, on peut tout de même penser à un conflit entre deux partis ou à un surcroît de méfiance envers des groupes qui pourraient ouvrir secrètement les portes de la ville. Autre disposition, la mise en défense de Mantes relève de précautions prises contre des stratégies militaires dont on connaît l'existence mais ignore le contenu. Il s'agit alors d'armer les habitants, d'ordonner aux quarteniers de faire le guet dans la ville et aux portes de jour comme de nuit, et aussi de placer un homme sur une tour de la collégiale Notre-Dame pour qu'il surveille les alentours et prévienne toute surprise ${ }^{38}$.

\footnotetext{
${ }^{36}$ DOUET-D’ ARCQ, Louis, Choix de pièces inédites..., op. cit., t. 1, p. 392-393.

${ }^{37}$ Mantes-la-Jolie, Archives Municipales, BB 3, fo $39 \mathrm{r}^{\circ}$ et $\mathrm{f}^{\circ} 30 \mathrm{r}^{\circ}$. Chaque année lors de l'élection du maire et prévôt de la ville et châtellenie de Mantes, les douze «pers et compaignons » se répartissent certaines missions — garder les chartes, exercer la juridiction de la prévôté ou encore garder les clefs des portes. Le cas évoqué ici n'est pas unique. Ce registre de délibération mentionne qu'à plusieurs reprises, les clefs ont été confiées à un ou deux hommes, souvent sur ordre ou avec l'assentiment du capitaine de la ville.

${ }^{38} I d$., $\mathrm{BB} 3, \mathrm{f}^{\circ} 49 \mathrm{v}^{\circ}$ (vendredi 3 février 1418), $\mathrm{f}^{\circ} 24 \mathrm{r}^{\circ}$ (vendredi 8 juillet 1418), $\mathrm{f}^{\circ} 15 \mathrm{v}^{\circ}$ (mardi 24 mai 1418).
} 
À Paris comme à Mantes, l'ensemble des mesures de prévention prend alors en compte l'existence d'une menace dont un certain nombre d'événements et le contexte immédiat viennent rappeler le danger.

Le secret n'est donc pas qu'une pratique anodine de cette guerre. La question de la révélation du secret - entendue comme moment et action permet d'aborder cette période non pas par la petite histoire mais par les jeux et les enjeux de la communication et de la politique. Il y a dans la publicité du secret une volonté de connaître et faire connaître un projet dissimulé et aussi, par moments, d'utiliser cette publicité et cette connaissance comme outil politique. Le secret entre donc souvent dans le champ de la propagande voire de la rumeur, surtout quand le secret révélé est seulement supposé.

Enjeux de pouvoir, les pratiques du secret doivent être contrôlées, qu'ils s'agissent des secrets du roi ou encore de la préparation de complots. Ce contrôle semble être confronté à une volonté répandue de savoir — qui va de la curiosité à l'espionnage —, volonté qui contribue à le justifier. L'espace public des villes est bel et bien investi par le secret au début du $\mathrm{XV}^{\mathrm{e}}$ siècle.

On pourrait enrichir sans doute cette étude très générale par une lecture croisée de sources diverses - chroniques, ordonnances royales ou encore lettres de rémission. L'espionnage et les espions, notamment, pourraient être alors mieux perçus. Quoi qu'il en soit, le secret reste une pratique et une réalité si répandues dans les sociétés humaines qu'il est toujours possible - à condition d'en contextualiser les formes — de trouver matière à en dévoiler non seulement le contenu, quand cela est possible, mais aussi, et là dans tous les cas, les mécanismes. 